Fecha de recepción: abril 2020

Fecha de aceptación: mayo 2020

Versión final: junio 2020

\section{Teorías del saber y el hacer de la enseñanza del diseño industrial en Guatemala.}

Gloria Carolina Escobar Guillén ${ }^{(1)}$

Resumen: El diseño industrial es una disciplina relativamente nueva en Guatemala, la primera universidad en impartir dicha carrera fue la Universidad Rafal Landívar en 1987. Los parámetros y metodologías de la enseñanza del diseño industrial destacan una gran influencia europea, que se debate entre dos corrientes: una de la Staatliche Bauhaus o Escuela de la Bauhaus y la otra de la Hochschule für Gestaltung-HfG de la Ulm. La primera fundada en 1919 en Weimar, Alemania por Walter Gropius, y la segunda inicia en 1953 en Ulm, Alemania por Otl Aicher, Max Bill e Inge Aicher-Scholl; ambas fundadas post primera y segunda guerra mundial, respectivamente. El objetivo de este trabajo es el de exponer ambas teorías y generar un análisis de cómo estás tienen relación con los enfoques temáticos para la enseñanza del diseño industrial en la URL, para fundamentar la o las corrientes que rigen su actual enfoque de enseñanza de la disciplina.

Palabras clave: Historia - Enseñanza - Diseño Industrial - Campo disciplinar.

[Resúmenes en inglés y portugués en la página 144]

(1) Licenciada en Diseño Industrial (URL), Posgrado en Ingeniería de Negocios (UGAL), Maestría en Reingeniería y Tecnologías de Aseguramiento (UGAL), becaria de la Maestría en Eco diseño y Eco innovación (UNICAM-Italia). Estudiante del Doctorado en Proyectos (UNINI-México), tesis Doctoral relacionada con el quehacer del diseñador industrial guatemalteco. Actualmente es investigadora y coordina el Subprograma en Diseño Industrial del Instituto de Investigación y Estudios Superiores en Arquitectura y Diseño (INDIS) de URL.

Dentro de las investigaciones que se coordinan en el Subprograma en Diseño Industrial del Instituto de Investigación y Estudios Superiores en Arquitectura y Diseño (INDIS) de la Universidad Rafal Landívar (en adelante URL), se encuentra la de El quehacer del diseñador industrial en Guatemala; la cual tiene por objetivo reconstruir el registro histórico de los primeros 30 años de la carrera de diseño industrial en Guatemala, enfocado en la identificación del proceso de evolución de la inserción laboral y del perfil del profesional 
egresado landivariano, y al mismo tiempo, en dimensionar el estado del sector en términos cuantitativos y cualitativos.

A continuación, se discutirán las teorías sobre un tema que es parte de dicha investigación: la influencia de la enseñanza del diseño industrial en Guatemala, de gran importancia debido a que interviene en la futura práctica profesional del diseñador industrial.

En el año 2017 se cumplieron 30 años de la carrera de diseño industrial en Guatemala, siendo la URL, la primera universidad en poner en marcha dicha carrera en el año de 1987, la cual empezó con una carrera a nivel técnico para posteriormente convertirse en licenciatura en el año 1991.

Por mucho tiempo el diseñador industrial se percibió como un diseñador de objetos y no se aceptaba que fuese un desarrollador o gestor, hasta que desde hace algunos años el mercado laboral les ha demandado hacerlo en Guatemala, así como internacionalmente se ha visto un cambio en el desempeño de dicho profesional. El diseño industrial en el país, se incorpora a la oferta de programas académicos en la década de los años ochenta en la primera universidad privada del país a nivel de técnico universitario. Morales (2019), en su tesis doctoral, expone:

El campo disciplinar del Diseño Industrial en Guatemala se institucionalizó como carrera universitaria en 1986, en la actual Facultad de Arquitectura y Diseño de la Universidad Rafael Landívar (URL). [...] En sus inicios, a partir de 1987, el programa de Diseño Industrial se ofreció como un técnico universitario, cuya primera cohorte egresó a finales de 1990. En el año 1993, el programa se reformuló como una licenciatura, cuyos primeros egresados datan de 1995. [...] Según los registros de la URL, en el año 2016 se graduaron 21 técnicos universitarios en Diseño Industrial y 323 licenciados en Diseño Industrial (URL, 26 de octubre de 2016), y se aprobaron 323 tesis de grado (p. 7).

El proyecto fue elaborado por la comisión de diseño industrial de la Facultad de Arquitectura, hoy Facultad de Arquitectura y Diseño, por el Arq. Daniel Borja Rosales, Ing. José Antonio Contreras Godoy y el diseñador industrial Sergio Rivera Conde (asesor) (Acta Consejo Directivo N. ${ }^{\circ}$ 3-87 Anexo A, 1986, p. 11). Presentado ante Consejo Directivo de la universidad, dicho equipo coordinado por el Arq. Borja (Acta Consejo de Facultad N. ${ }^{\circ} 18$ 86, quinto), elaboró la propuesta de carrera en el documento Proyecto de diseño industrial en el Anexo "A" de las actas de consejo directivo de la URL, donde se expone el concepto (proyectado) del perfil general del diseñador industrial:

El Diseñador Industrial, es un intérprete de las necesidades de su época, que conoce su país, su cultura, estudia y aporta soluciones a las demandas actuales y futuras en su campo, a través de conocimiento y aplicación de los recursos y la tecnología de su medio [...] usa el Diseño como disciplina de servicio, con el objetivo de lograr factores que en gran volumen son requeridos para el desarrollo comunitario; hacer el diseño más útil socialmente y no ponerlo al servicio de intereses mercantilistas [...] dentro de un grupo interdisciplinario 
sintetiza en forma creativa e innovadora las exigencias de uso para dar una respuesta proyectual específica (1986, p. 21).

Lo que se afirmaba en 1986, es que la misión de apertura de una carrera como ésta en la URL, era la de ser de beneficio para la sociedad, brindando mejoras a los involucrados, visualizándola como una carrera de desarrollo social. El arquitecto Daniel Borja citado por De León (2006) describió al diseñador guatemalteco como:

Un manejador de sistemas y un gestor de proyectos. Con calidad profesional del manejo del diseño [...] Podemos desarrollar una industria en base a sistemas propios, porque el diseño industrial no es simplemente el diseño de un objeto sino es el diseño de un sistema objetual, es la concepción de las líneas de producción del producto conociendo la calidad que debe de tener el producto, aportando e influenciando para que se maneje una industria manufacturera de mejor calidad y que responda de mejor manera a nuestras necesidad centroamericanas (p. 34).

La definición de Borja expone al diseñador industrial con sus competencias específicas y cómo éste aporta a las necesidades actuales, al contrario de De León que advierte en dónde puede ejercer. Ambas, se complementan brindando un perfil cualitativo de la época, de dicho profesional.

En el documento Enfoque temático para la enseñanza del diseño industrial 2010-2014 de la URL (2013), Szarata afirma:

Es importante notar que la disciplina es una actividad creativa, por tanto, se hace evidente la necesidad de desarrollar la creatividad en los estudiantes, para que lleguen a generar conceptos con un enfoque realmente innovador. Es importante mencionar que el diseñador industrial trabaja de manera multidisciplinar con otros profesionales [...] el diseñador desarrolla su profesión en un contexto de relaciones de trabajo conformado por profesionales de diferentes áreas, y tiene la responsabilidad de integrar todos los criterios relevantes determinados por el grupo [...] (p. 14).

Szarata define al diseñador como alguien que debe ser creativo y multidisciplinario, por lo que la academia destaca la necesidad en ser quien brinde las herramientas y experiencias necesarias para formar a los estudiantes durante su formación de pregrado. Además, el documento mencionado busca ser una guía para docentes y estudiantes, donde se encuentran las definiciones de los ejes para la enseñanza en diseño industrial, a través de una sistematización de los cursos de proyectos de la licenciatura: fundamentos del diseño, proyecto 1 , proyecto 2 , proyecto 3 , proyecto 4 , proyecto 5 , proyecto 6 , práctica profesional, proyecto de grado. En cada uno se explican: antecedentes, descripción del curso, objetivos, tendencias y los ejercicios realizados. (Szarata en URL, pp. 14-15) Dicho documento expone de una forma muy completa el eje proyectual de la carrera durante el año 2010-2014 (aún no se conoce una publicación de edición más reciente). 
El antecedente más reciente es de la diseñadora industrial Andrea Cano quien trabajó una investigación para el Subprograma de Diseño Industrial del Instituto de Investigación y Estudios Superiores en Arquitectura y Diseño (Indis) de la URL, la cual es insumo a la presente investigación y de donde Andrea Cano partió para desarrollar su proyecto de graduación de pregrado. Cano, en su tesis (2019), concluye que: es difícil definir el quehacer de un diseñador industrial, debido a que el alcance que tiene la carrera es amplio, pero es más sencillo describir el perfil de profesional, el cual define como:

Un DI es aquella persona preparada para investigar y tomar toda la información relacionada con un proyecto, digerirla, analizarla y proponer soluciones holísticas y estratégicas que resuelvan de la mejor forma posible las problemáticas planteadas inicialmente. Estas soluciones se pueden presentar como productos (que es la forma convencional de comprender la disciplina), servicios, experiencias y muchas otras propuestas que se proponen conforme al problema (p. 155).

Más de 30 años después, en el año 2019 se aprueba la nueva malla curricular 19001 en la que se generan cambios en la duración de la carrera, nombres de cursos, contenidos, entre otros. Como la descripción de la carrera en diseño industrial en la web de la URL afirma:

[...] ha creado un plan académico que se basa en un 'Modelo de integración progresiva'; de esta forma se sistematiza la educación y se logra que los estudiantes tengan una preparación completa e integral en los diferentes campos de acción, utilizando el diseño y la innovación para impulsar el desarrollo y mejorar la calidad de vida de las personas [...] el programa de estudios facilita en los futuros profesionales, habilidades y herramientas que incluyen una visión enfocada en la eficiencia para la utilización de recursos, la función y la estética de las soluciones, así como un amplio conocimiento de materiales y procesos de fabricación; desde una perspectiva general de la gestión y el emprendimiento a través del análisis del entorno social, económico y tecnológico (Universidad Rafael Landívar, s.f., parr. 4).

Además, agrega que al finalizar: "Sabrá identificar, evaluar y responder a las necesidades físicas y psicológicas de los usuarios mediante el desarrollo de productos y soluciones factibles de implementar o producir, viables y deseables" (URL, s.f., parr. 7).

Entonces, se puede decir que la más reciente definición del departamento de diseño industrial publicada en el sitio web de la URL define que un diseñador industrial, al emprender su camino profesional, antepone las necesidades del usuario, desarrolla productos factibles satisfaciendo necesidades y mejora la calidad de vida de la persona.

La enseñanza del diseño industrial en Latinoamérica se fundó en los modelos europeos del siglo veinte, específicamente con influencia de la Escuela de la Bauhaus y de la Hochschule für Gestaltung Hfg o Escuela de Ulm. A continuación, se expondrán las concepciones y teorías de ambas escuelas, se discutirán para llegar a conclusiones en base al análisis expuesto, y cómo se relaciona con la enseñanza del diseño industrial en Guatemala. 
La Escuela de la Bauhaus o Staatliches Bauhaus (Casa de la Construcción Estatal) fue la primera escuela de diseño, fundada por el arquitecto Walter Gropius en 1919 en Weimar, Alemania. Al finalizar en 1918 la Primera Guerra Mundial, se funda la República de Weimar en una Alemania derrotada. La Bauhaus se desarrolla en tres etapas, la primera en Weimar de 1919 a 1925, la segunda en Dessau de 1925 a 1932 y la tercera en Berlín de 1932 a 1933, hasta su cierre. En 1937 se funda La nueva Bauhaus en Chicago, Estados Unidos, continuando la línea de la Bauhaus. Gropius expone sobre la constitución de la escuela, citado por Sainz (2018):

Con el consentimiento del gobierno, denominé al nuevo instituto nacido de esta fusión 'Bauhaus Estatal de Weimar'. El objetivo fundamental de la constitución de la Bauhaus fue la síntesis de toda creación artística en una unidad, la conjunción de todas las disciplinas artísticas y técnicas como indisociables elementos constitutivos de una nueva arquitectura (p. 303).

En palabras de Walter Gropius “[...] surge la noción de una nueva unidad del mundo [...] la artesanía -y sobre todo la industria- comenzó a involucrar al artista un anhelo de belleza formal exterior [...]" (Sainz, 2018, p. 193 y 195). Donde se reúnen artes, sucesos, tendencias que no pueden separarse. La propuesta sobre la que se empezó a trabajar fue:

La Bauhaus tiene como objetivo la reunión de todo el quehacer artístico y la reunificación de todas las disciplinas del taller artístico en una nueva arquitectura, de modo que sean elementos indisociables. El fin último, aunque remoto, de la Bauhaus es la obra de arte unitaria (el gran edificio), en la que no hay límite entre arte monumental y decorativo (Sainz, 2018, p. 197).

En 1920 se crea el curso básico que era de inducción general, en el que se “[...] desarrolló un lenguaje visual abstracto y abstrayente que pretendía proporcionar una base teórica y práctica para cualquier empresa artística" (Abbott y Lupton, 2019, p. 11) El curso básico enseñaba los principios fundamentales a los estudiantes sobre formas y materiales. Los estudiantes se seleccionaban si en el curso preliminar destacaban según los trabajos presentados y su talento. Vale mencionar que en esta etapa los trabajos no eran colectivos.

El curso básico estaba formado por el trabajo en el taller, alternando con varios oficios, y con guía técnica del superior en el taller. Los cursos que se ofrecían en esta etapa constaban de: dibujo natural, dibujo técnico y construcción, matemáticas, física y mecánica; además teoría sintética del espacio y teoría de la armonización (Sainz, 2018, p. 201). La Bauhaus apostaba porque el estudiante se formara con la instrucción del maestro artesano y la instrucción del maestro de la teoría, "Ambos mantienen un estrecho vínculo pedagógico. La Instrucción Artesanal y la Teoría de la Forma forman la base: ningún aprendiz u oficial puede quedar díspensado de una o de la otra” (Sainz, 2018, p. 202).

Hasta 1923, el curso básico lo impartió Johannes Itten, que poseía experiencia en técnicas educacionales fundamentadas en la infancia. "Itten buscaba liberar la creatividad de los estudiantes mediante un retorno a la infancia, introduciendo exploraciones elementales 
de formas y materiales, el automatismo, el dibujo a ciegas, movimientos rítmicos de dibujo y un enfoque intuitivo y místico" (Abbott y Lupton, 2019, p. 26).

El diagrama de cursos de la Bauhaus de 1922 es característico por su presentación de anillos concéntricos: el primer anillo trata de la enseñanza preliminar con duración de medio año, como resultado ingresaban a un taller para ser aprendices; el segundo y tercer anillo constan de los cursos de: teoría de los materiales y de los utensilios, estudio de la naturaleza, teoría del espacio, teoría del color, teoría de la composición y teoría de las construcción y la representación, además de los cursos de taller práctico en talleres de oficios donde aprendían materiales, tales como: piedra, madera, metal, tejidos, color, vidrio y arcilla, con una duración de 3 años; el anillo central es para la Bauhaus el más relevante, el núcleo, considerado como el de construcción, donde se experimenta el proyecto, la duración del núcleo central depende del desarrollo, las capacidades y la coyuntura. La posición de la construcción en el centro hace eco al manifiesto fundacional de Gropius, donde dice que el "último fin de todos las artes visuales es la construcción completa" (Abbott y Lupton, 2019, p. 11). "La obra y el campo de experimentación, en intercambio recíproco, dan continuidad a la Instrucción artesanal y a la Teoría de la forma. Resultado: título de maestro otorgado por la Cámara de Artesanos y, llegado el caso, por la Bauhaus” (Sainz, 2018, p. 198). La Bauhaus acepta la máquina como el medio más moderno de la creación y busca entenderse con ella. Pero no tendría sentido enviar a la industria al aprendiz dotado para la creación sin una preparación artesanal, confiando en restablecer así la relación con el mundo laboral [...] (ídem, p. 202). La Bauhaus no quiere retornar al antiguo artesanado, busca vincularse con la industria. Pero, sabe que sus estudiantes deben relacionarse con los oficios, conocerlos y practicarlos, para poder llegar a desempeñarse en las empresas industriales y potencializar sus conocimientos técnicos. "[...] la enseñanza de la Bauhaus, que comprende el ámbito artesanal y el científico de la creación artística” (Sainz, 2018, p. 197). La teoría de la Gestalt o teoría de la forma fue una de las más dominantes en la enseñanza del diseño. Los ejercicios se fundamentan en la observación estudiando la naturaleza y generando abstracciones o analogías para su composición. Además, la representación o concepción espacial, donde se apoyan con la generación de maquetas. Es con la Bauhaus que se deja atrás el dibujo con punto de fuga y saltan hacia el dibujo técnico o geométrico que mantiene la génesis natural (Sainz, 2018, p. 206). La Escuela destacó por dar a conocer sus resultados en exposiciones, entre ellas la de Weimar en 1923 llamada 'Arte y técnica, una nueva unidad', lo que permitió dar a conocer sus trabajos prácticos enfocados en el “desarrollo y resolución de problemas" (Sainz, 2018, p. 305).

En 1933 se ordena el cierre de La Bauhaus en Berlín. Varios de los alumnos y maestros huyen del país y se trasladan a Estados Unidos. En Chicago, László Moholy-Nagy crea la Nueva Bauhaus, el Instituto de Diseño de Chicago.

Años más adelante, durante seis años se desarrolló la segunda guerra mundial. La eliminación de dicha contienda generó que muchas industrias quedaran destruidas, esto benefició en la necesidad de innovación para reactivarse desde cero, en materia de tecnología y producción. Es en el año de 1950 que se funda La Hochschule für Gestaltung de Ulm, una de las escuelas más destacadas de la época, en educación en diseño que implementa metodologías de enseñanza. 
El origen de este proyecto nace con Inge Scholl y su esposo Otl Aicher, para rendir homenaje a los dos hermanos pequeños de Scholl que fueron ejecutados durante la guerra.

En 1946 fundaron la Volkshoschule de Ulm, un centro educativo para la enseñanza de personas adultas [...] En 1947 comenzaron a pensar en la apertura de una escuela que tuviera entre sus objetivos contribuir a la restauración de una sociedad destruida [...] Hacia 1949 habían dado ya forma a la idea de crear una nueva institución educativa centrada en la política y en el periodismo pero que incluiría otras disciplinas relativamente afines como la fotografía, el diseño industrial o el urbanismo (Vega Pindado, 2013, p. 4-5).

Existe un interés de Scholl y Aicher porque Max Bill dirija la escuela de diseño industrial para continuar con las ideas de la Bauhaus. Bill se convierte en director de la escuela y evidentemente la enseñanza continúa con las teorías bauhausianas.

En 1954 Bill invita al diseñador industrial argentino Tomás Maldonado, quien busca abandonar los antiguos métodos, pero Bill tiene el concepto de continuar con la Bauhaus. Maldonado, promueve que los diseñadores se enfrenten a la tecnología y a la industria; estas diferencias de pensamiento generaron controversia entre los métodos de enseñanza. En la escuela de Ulm se favorecen los trabajos interdisciplinarios, integración de nuevas materias teóricas, la creación de prototipos, pero principalmente el énfasis en la metodología del diseño (Vega Pindado, 2013, p. 6).

La estructura de estudios se plantea para que dure cuatro años, iniciando con el curso básico (1953-1956), muy parecido al curso fundamental de la Bauhaus, solo que en este caso la duración del curso inicial se propone de un año. A partir del segundo año existen cuatro especialidades "diseño de producto, comunicación visual, construcción y, hasta 1962, información” (Vega Pindado, 2013, p. 7).

El curso fundamental o Vorkurs Bauhausiano, propuesto por Max Bill compuesto por círculos que se intersectan, dentro de un círculo mayor, integraba materias de urbanismo, metodología política, prensa, información publicitaria, película fotográfica, diseño de producto y arquitectura. En el círculo de la periferia se aunaban cursos de: política, economía, filosofía, psicología, historia contemporánea y sociología. Maldonado llega a reformar el famoso curso básico, con materias fuera del ámbito artístico, con ello buscaba:

Introducir a los alumnos en métodos de análisis aplicados a problemas de diseño. Familiarizarlos con la nueva civilización tecnológica. Acostumbrarlos a trabajar como parte de un equipo. Equilibrar los niveles de formación tan dispares con que cada alumno llegaba a Ulm.

El estudio de las relaciones espaciales, las matemáticas o la teoría de la percepción ocuparon un lugar relevante. La semiología, entendida como teoría de los signos contribuyó al soporte pseudocientífico del currículum en esta nueva etapa donde la metodología tendría un papel relevante. (Vega Pindado, 2013, p. 7-8). 
La escuela de Ulm no tardó en ganar reconocimiento internacional, debido a que contaba con estudiantes fuera de Alemania, principalmente de Francia. Dichos estudiantes llevaron los conceptos al país francés, para continuar con las teorías de la HfG de Ulm.

La pedagogía de la enseñanza de la HfG destaca en dos modalidades: primero la de organización y segundo la de comunidad libre. La primera, se define como la de conferencia, autorizada, de control, el docente está al frente de la audiencia; la segunda, rol de asesor, aprendizaje experimental, enseñanza más libre, aprendizaje por medio de discusión y práctica, el docente gira en torno a grupos de trabajo, no tiene una posición fija (Côme, 2017, p. 5-6).

Con respecto a la especialidad del diseño industrial, que fue también llamada forma del producto, algunas de sus concepciones fueron: despreciar el diseño de productos de lujo, integrar factores funcionales, tecnológicos y culturales, además crece la importancia en explorar al usuario de los productos. En la HfG de Ulm se tenían talleres prácticos, pero en menor cantidad comparado con la Bauhaus, aunado a esto se involucraron cursos de sociología, filosofía y ergonomía. Sumado a esto se hicieron alianzas con el Instituto del diseño industrial, donde colaboraron con empresas, como Braun (Vega Pindado, 2013, p. 8). En 1958 crean las llamadas agencias de diseño o grupos de desarrollo, donde los estudiantes trabajaban para empresas, generando vínculos y diferenciando la enseñanza de la escuela con la práctica en la industria, algo semi-profesional.

[...] la relación entre teoría y práctica en el plan de estudios se hizo más equilibrada y se convirtió en un modelo imitado por otros centros. Por otra parte algunas empresas vieron la posibilidad de poner en práctica sistemas de producción cercanos a estos planteamientos como en el caso de Braun y su acercamiento al "Buen diseño" (Vega Pindado, 2013, p. 10).

Con la salida de Max Bill, Tomás Maldonado asume la dirección de la Escuela, su concepto residía en alejarse de los conceptos iniciales, convertirse en el diseñador que toma decisiones en la industria, y ya no en el antiguo artista con aire de superioridad. A continuación, se expone un discurso de su autoría de 1958, citado por Vega Pindado (2013):

La HfG hace suya la tesis según la cual, el proyectista, aun trabajando para la industria, ha de continuar asumiendo sus responsabilidades frente a la sociedad. En ninguna circunstancia sus obligaciones para con la industria podrán anteponerse a sus obligaciones con la sociedad. Se ha de propiciar la formación de un nuevo tipo de proyectista que, en las actuales y difíciles condiciones de la sociedad capitalista, sepa crear objetos concebidos al margen de cualquier oportunismo o profesionalismo (p. 10).

Es claro el acercamiento al proceso científico y por otro lado, se aleja de la intuición y la estética. En la década de los 60’s se incrementa la enseñanza académica. (Vega Pindado, 2013, pp. 10-11). La influencia de la enseñanza de la HfG en Latinoamérica es evidente, tal como DeValle (2016) expone: 
La Escuela de Ulm ha influido notoriamente en el desarrollo del diseño en Latinoamérica. Prueba de ello es la incorporación de metodologías ulmianas en los procesos de diseño, en la comprensión de estos como disciplinas científicotecnológicas en gran parte de Sudamérica (Brasil, Chile, Argentina, Uruguay). Probablemente ambas características surgen en la temprana llegada -fines de los 50 y comienzos de los 60 del siglo $\mathrm{xx}$ - de las ideas ulmianas a centros universitarios donde se comenzaban a impartir cursos de diseño. Este hecho ha alimentado la idea de que ese intercambio se originó en ese contexto y que se trató de una rareza o de un absoluto pionerismo de arquitectos y artistas latinoamericanos (p. 53).

La institucionalización de la carrera de diseño industrial en Guatemala se remonta a la década de 1970, aunque en su momento no tuvo ingreso de estudiantes, ésta nuevamente se presenta en la década de los años 80, para abrir el técnico universitario en 1987 en la primera universidad privada del país, la Universidad Rafael Landívar.

El plan de estudios del año 1975 buscaba el desarrollo a través de la industria, pero buscando el bien común de la sociedad, entendiendo las necesidades del consumidor y considerando la realidad nacional. En ese momento la carrera se llamaba Técnico Universitario en Desarrollo de Producto (Diseño Industrial) tenía una fuerte base de Ingeniería y de Arquitectura, en ese entonces el departamento de Arquitectura se encontraba dentro de la facultad de Ingeniería. El primer año del plan de estudios se proponía que los dos semestres fueran en común con Arquitectura, el segundo año se planteó que los cursos fueran en común con Ingeniería, pero ya se visualizaba la propuesta de tener clases únicas de diseño, para la carrera de diseño industrial. En el quinto semestre denota una carga más fuerte en clases específicas de diseño industrial, así como los laboratorios y talleres que serían concretos para dicha carrera técnica (URL, 1975, pp. 295-296).

Lo que podemos destacar en este primer plan de estudios es que se toma la influencia del curso fundamental de la Bauhaus, pero con duración de la Hgf de Ulm de un año, el cual se proponía realizar en común con Arquitectura donde existían los cursos de antropología, lenguaje, teoría del arte, matemáticas, dibujo técnico, expresión gráfica, sociología, psicología, historia del arte y la cultura, geometría descriptiva y diseño. Pero a la vez, destacan pocos talleres prácticos un poco más influenciado por la HfG de Ulm, los laboratorios de materiales propuestos en ese plan de estudios fueron de metales, cerámica, madera y textiles. La iniciativa resurge en el año de 1986, en donde el pensum de estudios estuvo influenciado en gran parte por Universidad Autónoma de México (UAM) y en la docencia se aúnan catedráticos de Colombia. Se contó con un nuevo plan de estudios, coordinado por el Arq. Daniel Borja, Ing. José Antonio Contreras y como asesor Diseñador Industrial Sergio Rivera de México. El plan de estudios a nivel técnico de la carrera de diseño industrial donde se denota el sistema de eslabones (de la UAM) y el crecimiento gradual en secuencia horizontal de los cursos, iniciando con un nivel introductorio en el primer semestre -como en la Bauhaus-, en este caso contenía los cursos de: historia del arte I y el diseño, lenguaje, diseño fundamental, matemática I, comunicación I (expresión gráfica I y dibujo técnico I). Continuando con un nivel básico en el segundo y tercer semestre, estructurado de los cursos de: historia y arte del diseño II y III, ergonomía, taller I y II (diseño formal, 
metodología del diseño), física general, matemáticas II y III, estática, comunicación II (expresión gráfica II y dibujo técnico II) y técnicas de presentación I. Seguidamente en los próximos tres semestres consta del nivel técnico, integrado por los cursos de: estudio sociocultural de Guatemala, ecología, ética profesional, taller III (diseño industrial II y materiales y procesos I, resistencia de materiales), taller IV (diseño industrial III, materiales y procesos II y mecanismos) y taller V (diseño industrial IV, materiales y procesos III), técnicas de presentación II, principios económico administrativos I y II, computación. Finalizando por un último semestre representando el nivel de cierre con: seminario de diseño industrial I y la práctica profesional; se destaca la conexión de contenidos en la relación vertical de cada semestre (URL, 1987, anexo A punto 10).

El plan curricular de 1986 destaca características mayormente Ulmianas, al orientarse más a lo científico y menos a lo artístico, incluso el acercamiento de la carrera en el último semestre con la práctica profesional, denota algo característico de la Escuela de Hfg de la Ulm, al tratar de llevar al plano semi-profesional a los estudiantes de su escuela y acercarse a la industria.

En 1989 se integran al cuerpo docente de la carrera en diseño industrial de la URL, los colombianos Cielo Quiñonez, William Vásquez y Gabriel García, quienes fueron formados por alemanes bajo la corriente Bauhaus, lo que inyectó parte de ello a la didáctica y que se reflejó en proyectos aplicando la practicidad de los oficios y la semiología con la modernidad guatemalteca, generando una nueva identidad del diseño.

Más adelante se crearon vínculos más fuertes entre los estudiantes de diseño con la industria, una concepción fuertemente ligada a Ulm, como por ejemplo un lazo que aún se mantiene es la relación con la Asociación de Exportadores de Guatemala (AGEXPORT), además los proyectos y prácticas profesionales con Micro, pequeñas y medianas empresas (Mipyme) del país.

El departamento de diseño industrial de la URL, después de la puesta en marcha de la carrera ha realizado seis renovaciones curriculares, hasta llegar a la más reciente, el número 19001 del año 2019, la cual ha sido la renovación más grande. Este nuevo pensum, promete la duración de cuatro años, en tres ejes temáticos: a. Humanística, b. Proyectual y c. Tecnológica, y ocho áreas específicas: a1. Fundamentos teóricos del diseño, a2. Factores humanos e investigación, b1. Diseño, b2. Gestión del diseño, b3. Comunicación y expresión, c1. Producción, c2. Materiales y producción, y c3. Tecnología. Dentro de los cambios más relevantes se encuentra el interés por aumentar la investigación durante la carrera, así como fortalecer las áreas de administración, marketing y finanzas para el diseño, eliminar los cursos de historia del arte para enfocarse a historia del diseño industrial, cambian los nombres de los cursos de diseño que por mucho tiempo se llamaron "proyecto" ahora vuelven a su naturaleza original "diseño industrial".

La enseñanza landivariana para el diseño industrial se puede concluir que tiene rasgos tanto bauhausianos como ulmianos. Aunque, pesa más la corriente ulmiana, esto por la educación por medio de metodologías del diseño, consideración del usuario y la investigación como fuerte aspecto dentro del proceso de diseño, vinculación con la industria y las empresas a modo de obtener una experiencia semi-profesional. $Y$ en menor escala, el concepto bauhausiano, debido al interés por resolver necesidades de la sociedad, modernizar el diseño, vincular los oficios como base de conocimiento para emprender en la industria. 
Se concluye con unas interrogantes, que buscan ser provocativas a abrir nuevos planos de discusión en el ámbito de la enseñanza del diseño industrial. Ahora, en medio de una pandemia a causa del Covid19, ¿cómo, la situación actual mundial, influirá a generar nuevas formas de enseñanza del diseño? ¿Crearán nuevas metodologías de enseñanza que en un futuro serán las teorías que se discutirán como nuevas identidades y corrientes de la didáctica del diseño industrial?

\section{Listado de referencias bibliográficas}

Cano, A. (2019). Feedback for all: modelo de obtención de retroalimentación para el departamento de diseño industrial. (Tesis de licenciatura). Universidad Rafael Landívar: Guatemala.

Côme, T. (2018). De la Hochschule für Gestaltung de Ulm al Institut de l'Environnement de París: revoluciones en la enseñanza de la arquitectura y el diseño durante los años 1960. Trad. por A. Ávila, y D. C. Ruiz, D. C. Apuntes, 31(2). Recuperado de http://doi.org/10.11144/ Javeriana.apc31-2.hgui

Cultier. (s.f.) Bauhaus, la primera escuela de diseño del siglo XX. Recuperado de: http://www. cultier.es/bauhaus-la-primera-escuela-de-diseno-del-siglo-xx/

De León, M. (2006). Historia del Diseño Industrial en Guatemala y análisis comparativo de las temáticas de Proyecto, con la evolución histórica del Diseño en el mundo proyectado hacia el enfoque del diseñador industrial guatemalteco actual. (Tesis de licenciatura). Guatemala: Universidad Rafael Landívar.

Devalle, V. (2016). América Latina, la otra sede de la hfg-Ulm. RChD: creación y pensamiento, 1(1), pp. 53-63. doi: 10.5354/0718-2430.2016.4420 Recuperado de: https://rchd.uchile. cl/index.php/RChDCP/article/view/44202

Lupton, E. y Abbott, J. (eds.). (2019). El abc de la Bauhaus y la teoría del diseño. Barcelona: Editorial Gustavo Gili. ISBN: 978-84-252-3265-7 (PDF digital).

Morales, H. (2019). La institucionalización del diseño industrial durante la década de 1980. La apuesta jesuita por el diseño industrial como una alternativa para el desarrollo económico de Guatemala, desde los sectores agroindustriales y artesanales. (Tesis de doctorado). Buenos Aires: Universidad de Palermo.

Sainz, J. (Dir.). (2018). Walter Gropius: proclamas de modernidad: escritos y conferencias, 1908-1934. Barcelona: Editorial Reverté. Recuperado de: https://elibro.net/es/ereader/ rafaellandivar/105170?page $=4 \mathrm{E}-\mathrm{ISBN}$ : 9788429194654

Universidad Rafael Landívar. (2013). Enfoque temático para la enseñanza del diseño industrial 2010-2014. Guatemala: Editorial Cara Parens.

. (s.f.) Licenciatura en Diseño Industrial. Recuperado de https://principal.url. edu.gt/index.php/carreras/facultades/facultad-de-arquitectura-y-diseno/licenciaturaen-diseno-industrial . (1975, 10 diciembre). Consejo Directivo Acta N. ${ }^{\circ}$ 19-75. Archivo de la Secretaría General de la Universidad Rafael Landívar. 
(1987, 18 febrero). Consejo Directivo Acta N. ${ }^{\circ}$ 3-87. Archivo de la Secretaría General de la Universidad Rafael Landívar.

Vega Pindado, E. (2013.) HfG Ulm. El diseño en la Alemania del Wirtschaftswünder. infolio $\mathrm{n}^{\circ}$ 1. ISSN 2255-4564. Recuperado de: http://www. infolio.es/articulos/vega/ulm.pdf

\begin{abstract}
Industrial design is a relatively new discipline in Guatemala, the first university to teach such a career was the Rafal Landívar University in 1987. The parameters and methodologies of teaching industrial design highlight a great European influence, which is debated between two currents : one from the Staatliche Bauhaus or Bauhaus School and the other from the Hochschule für Gestaltung-HfG in Ulm. The first one founded in 1919 in Weimar, Germany by Walter Gropius, and the second one started in 1953 in Ulm, Germany by Otl Aicher, Max Bill and Inge Aicher-Scholl; both founded post first and second world war, respectively. The objective of this work is to expose both theories and generate an analysis of how they are related to the thematic approaches for teaching industrial design at the URL, to support the current or currents that govern their current approach to teaching the discipline.
\end{abstract}

Keywords: History - Teaching - Industrial Design - Disciplinary field.

Resumo: $\mathrm{O}$ desenho industrial é uma disciplina relativamente nova na Guatemala, a primeira universidade a ministrar esse diploma foi a Universidade Rafal Landívar, em 1987. Os parâmetros e metodologias para o ensino do desenho industrial destacam uma grande influência européia, debatida entre duas correntes : um da Staatliche Bauhaus ou Bauhaus School e outro da Hochschule für Gestaltung-HfG em Ulm. O primeiro, fundado em 1919 em Weimar, Alemanha, por Walter Gropius, e o segundo iniciado em 1953 em Ulm, na Alemanha, por Otl Aicher, Max Bill e Inge Aicher-Scholl; ambos fundaram pós primeira e segunda guerra mundial, respectivamente. $\mathrm{O}$ objetivo deste trabalho é expor as duas teorias e gerar uma análise de como elas estão relacionadas às abordagens temáticas para o ensino do desenho industrial na URL, para apoiar as correntes ou correntes que governam sua abordagem atual no ensino da disciplina.

Palavras chave: História - Ensino - Desenho Industrial - Disciplina.

[Las traducciones de los abstracts fueron supervisadas por el autor de cada artículo] 